\title{
Condition Assessment of High Voltage Circuit Breaker Operating Mechanism Based on Coil Current Waveform
}

\author{
Xiaoli Duan ${ }^{1}$, Hongcai Lei ${ }^{1}$, Min Fan ${ }^{1}$, Lihua Zhao ${ }^{2,}$, Rongrong $\mathrm{Fu}^{2}$, Qiang Rong ${ }^{3}$, Hao Zhang ${ }^{4}$ and Zhong Wang ${ }^{2}$ \\ ${ }^{1}$ Hunan Electrical Power Research Institute, State Grid Corporation, 410000, Changsha, China \\ ${ }^{2}$ College of Electrical and Information, Sichuan University, 610065, Chengdu, China \\ ${ }^{3}$ Huawei Corporation, 610065, Chengdu, China \\ ${ }^{4}$ State Grid Corporation, 618000, Deyang, China \\ ${ }^{*}$ Corresponding author
}

\begin{abstract}
In order to evaluate the condition of the high voltage circuit breaker (HVCB) operating mechanism, a method based on the coil current (CC) waveform of the operating mechanism has been studied in this paper. A finite element method (FEM) with high accuracy has been used to establish an electromagnetic system model of the operating mechanism. The effects of the mechanical parameter variations and mechanism faults on the CC waveform have been studied by simulation and experimental studies, and the features and inflections of the $\mathrm{CC}$ waveform are determined as the evaluation indices of the mechanism. Then the slope method has been used to extract features and inflections of the CC waveform after filtering, and the CC features are optimized by Pearson correlation coefficient method. Finally, the condition of the HVCB operating mechanism has been evaluated by the combination of the waveform recognition and neural network analysis. It has been concluded that the evaluation results can accurately reflect the operation condition of the HVCB operating mechanism.
\end{abstract}

Keywords-coil current waveform; condition assessment; finite element method; feature and inflection extraction; high voltage circuit breaker; neural network; operating mechanism of circuit breaker; Pearson correlation coefficient; slope method; waveform recognition

\section{INTRODUCTION}

HIGH voltage circuit breakers (HVCBs) play an important role in the delivered power quality and reliability, as well as in the promotion of customer satisfaction. HVCBs are prone to mechanical failure. Relevant statistics have showed that $43 \%$ $45 \%$ of the major failure of HVCBs is caused by the operating mechanism. Therefore, the safety and reliability of the operating mechanism are important factors to ensure reliable operation of HVCBs [1]-[7].

The trip/close $\mathrm{CC}$ waveform contains much state information of the operating mechanism, which can reflect the working condition of the electromagnet of the operating mechanism, the latch or valve that is controlled by the electromagnet, the transmission mechanism of switch and the auxiliary contact during operation. Based on the condition assessment of the operating mechanism, we can timely learn and grasp the running state of the mechanism, and make reasonable maintenance plan, which is of great significance to ensure the safe operation of the power grid.

The condition assessment of the HVCB operating mechanism based on the $\mathrm{CC}$ has been investigated from various perspectives in the literature. For instance, the evaluation algorithm is established to evaluate the condition of the mechanism by analyzing the relationship between the features of the CC and the state of the mechanism [8], [9]. The effect of the state changes of the operating mechanism on the $\mathrm{CC}$ waveform can be well simulated and analyzed by using the magnetic circuit method to establish the electromagnetic system model of the mechanism [10]-[12]. The condition of the operating mechanism is evaluated by the support vector machine (SVM) based on the features of the CC waveform [13].

In view of all that has been mentioned so far, we believe that the CC plays a significant role in the condition assessment of HVCBs. And the above mentioned methods have gained a certain application, but they only considered the changes of the $\mathrm{CC}$ waveform features, but ignored the variations in the waveform itself apart from the overall features, which indicates that the assessment results failed to fully reflect the actual condition of the operating mechanism.

This paper focuses on exploring the features and inflections of the $\mathrm{CC}$ waveform as the evaluation indices to evaluate the condition of the HVCB operating mechanism. The results will provide a useful and practical insight into HVCB operating mechanisms condition. The proposed approach is clarified in the following way in this paper. Having explained the correlation between the CC and HVCB operations, the electromagnetic system model of the operating mechanism is established based on the FEM. The effects of mechanical parameter variations and mechanism failures on the CC waveform are studied by simulation and experimental studies to determine the features and inflections of the $\mathrm{CC}$ waveform as the evaluation indices of the mechanism. Furthermore, the features and inflections of the $\mathrm{CC}$ waveform are extracted by the slope method, and the features of the CC are optimized by using the Pearson correlation coefficient to avoid the "curse of dimensionality". Finally, the waveform recognition and neural network are combined to evaluate the 
operating mechanism of HVBCs. The assessment results can reflect the operation condition of the HVCB operating mechanism accurately.

\section{TRIP/ClOSE CC WAVEFORM}

Trip and close $\mathrm{CC}$ waveform of the operating mechanism is very similar, so this paper only analyzes the trip CC waveform. The typical trip $\mathrm{CC}$ waveform is shown in FIGURE I According to the typical current waveform of FIGURE I, the moving process of the electromagnet core can be divided into 4 stages.

Stage $t_{0} \sim t_{1}$ : At $t_{0}$ moment, the coil is energized. In this stage, the iron core does not move, and the CC is related to coil voltage, circuit resistance, iron core air travel and whether the iron core jams.

Stage $t_{1} \sim t_{2}: t_{1}$ moment, moving iron core begins to move. The CC waveform at this stage can reflect the fault of the iron core jam, the deformation of the impact rod or the failure of the release.

Stage $t_{2} \sim t_{3}: t_{2}$ moment, moving iron core stop moving. In this stage, the latch of transmission mechanism is opened by the moving contact action. The magnitude of the current $i_{3}$ reflects the condition of the coil voltage and the resistance of the circuit. The $\mathrm{CC}$ waveform at this stage can reflect the working condition of the transmission mechanism of the switch.

Stage $t_{3} \sim t_{4}: t_{3}$ moment, the auxiliary contact cut off the coil circuit power supply; $t_{4}$ moment, the $\mathrm{CC}$ drops to 0 . The $\mathrm{CC}$ waveform at this stage can reflect the working condition of the auxiliary contact.

Obviously, $t_{0}, t_{1}, t_{2}, t_{3}, t_{4}, i_{1}, i_{2}$ and $i_{3}$ are the key turning points to reflect the $\mathrm{CC}$ waveform and its the features. By analyzing the $\mathrm{CC}$ waveform and the features, the condition of the electromagnet and the main body of the operating mechanism can be well evaluated.

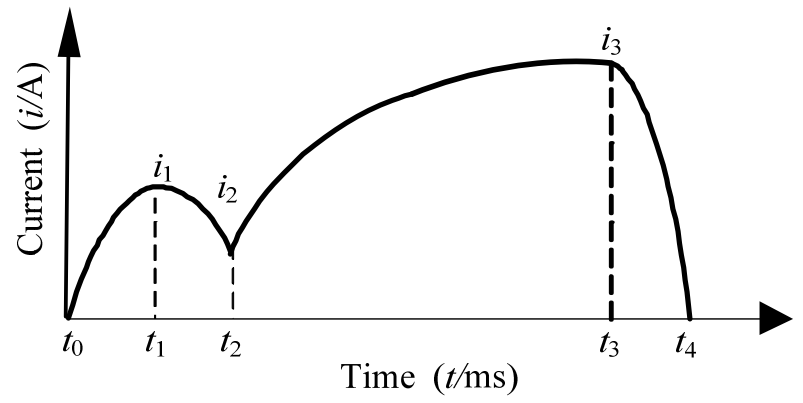

FIGURE I. STANDARD TRIP CC WAVEFORM.

When faults occur in the operation mechanism, the parameters related to the features of trip/close CC waveform may change. At the same time, the shape of the waveform may also change. For instance, if the main body of the operating mechanism occurs jam faults, the CC waveform at stage $t_{2} \sim t_{3}$ will appear inflections. These inflections are also important aspects to judge the condition of the operation mechanism.
Figure II presents the situation that each stage of the CC waveform contains inflections. As we can see from Figure II (a), $t_{0} \sim t_{2}$ stage appears inflections a and $\mathrm{b}$, which indicates that the moving iron core occurs jam fault. In Figure II (b), stage $t_{2} \sim t_{3}$ appears inflections $\mathrm{c}$ and $\mathrm{d}$, which indicates that the transmission mechanism of the switch doesn't move smoothly and occurs jam fault. In Figure II (c), stage $t_{3} \sim t_{4}$ appears inflections e and $\mathrm{f}$, which indicates that the auxiliary switch bounces or can-not be disconnected.

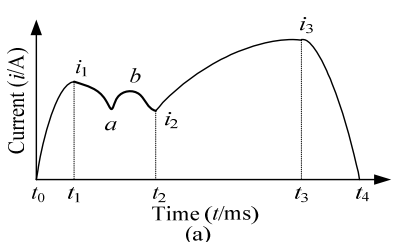

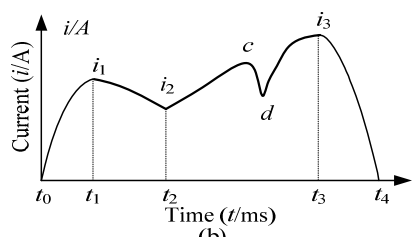

(b)

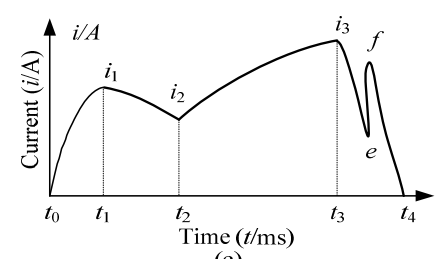

(c)

FIGURE II. THE TRIPPING CC WAVEFORM WITH THE INFLECTIONS

\section{The Model of Electromagnetic System Based on FEM}

The FEM has excellent applicability, and its application range is very wide. It is expressed in matrix form, so it is convenient to compile the computer software. Compared with the magnetic circuit method, it does not need to analyze the magnetic circuit in advance, and it can be directly used to analyze the magnetic field distribution. The FEM is simple. And it has higher calculation precision [14], [15]. Therefore, in this paper, the FEM is used to model the electromagnetic system, simulating and analyzing effects of the condition changes of operating mechanism on the $\mathrm{CC}$ waveform.

\section{A. The Motion and Circuit Equation of Electromagnetic System}

The motion process of the electromagnetic system can be analyzed by transient dynamics. The basic motion equation for transient dynamics is:

$$
[\boldsymbol{M}]\{\ddot{\boldsymbol{u}}\}+[\boldsymbol{C}]\{\dot{\boldsymbol{u}}\}+[\boldsymbol{K}]\{\boldsymbol{u}\}=\{F(t)\}
$$

Where, $[\mathrm{M}],[\mathrm{C}],[\mathrm{K}]$ are mass matrix, damping matrix and stiffness matrix, respectively. $\{\ddot{\boldsymbol{u}}\},\{\dot{\boldsymbol{u}}\},\{\boldsymbol{u}\}$ are acceleration vectors, velocity vectors and displacement vectors of the electromagnetic system moving nodes, respectively. $\{F(t)\}$ is a time-varying function of load.

The voltage equation of the coil circuit is: 


$$
U=I R_{c}+\frac{\partial \lambda(z, I)}{\partial t}
$$

Where, U , z, I, Rc are the DC voltage across the coil, the air gap length of electromagnetic system, the coil current and the coil resistance, respectively.

Based on the above analysis, the magnetic field and the movement of the electromagnetic system can be solved by the FEM. In addition, corresponding model can be established to calculate the relevant parameters of electromagnetic system according to the actual size of the structure and parameters of the electromagnetic system.

\section{B. The Calculation of Electromagnetic System \\ Characteristics Based on FEM}

The distribution of the magnetic field of the electromagnet in the two dimensional model are shown in Figure III. As is shown in Figure III, the magnetic flux is mainly distributed in the magnetic core, and there is a small part of leakage flux between the middle and the side of the core column. This is consistent with the actual magnetic field distribution of the operating mechanism electromagnet, which shows that the FEM can simulate the electromagnetic system of operating mechanism accurately and reliably.

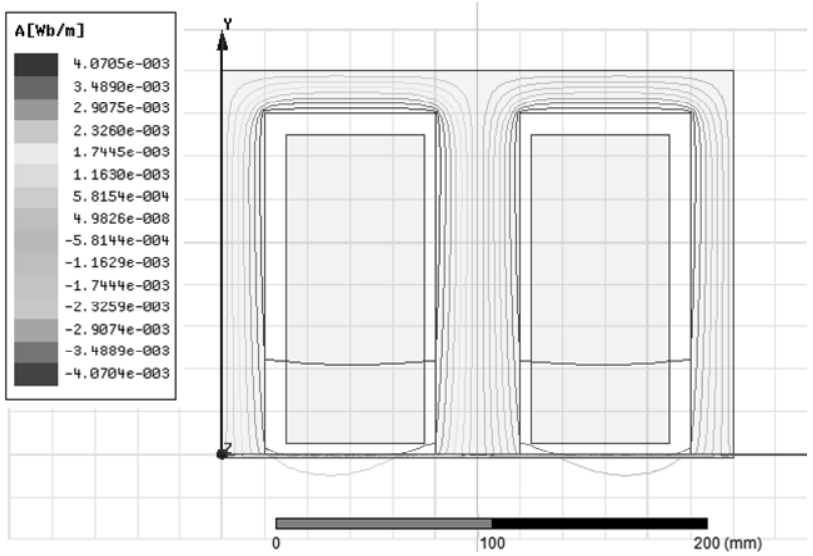

FIGURE III. DISTRIBUTION OF ELECTROMAGNET FIELD BASED ON FEM.

\section{ANALYSIS OF SimUlation AND EXPERIMENTAL STUDIES RESULTS OF THE CC WAVEFORM}

A type of $40.5 \mathrm{kV}$ HVCB spring operating mechanism is selected as the reference to study the influence of mechanical parameter variations and mechanism faults on the $\mathrm{CC}$ waveform, including coil voltage, circuit resistance and mechanism fault. In this paper, the trip CC as an example is explained in detail.

The simulation and actual measured waveforms of the CC when the coil voltage varies is shown in Figure IV. Figure IV indicates that the lower the coil voltage is, the slower the current rises at stage $t_{0} \sim t_{1}$ and the longer the duration of the current is, which means that the longer the inherent operation time of moving contact is, the longer the coil is powered. And we can see from Figure IV, each feature of the current is also reduced when the coil voltage is reduced. What's more, when the voltage is $65 \%$ of the rated voltage, the $\mathrm{CC}$ waveform has seriously deviated from the normal current waveform.

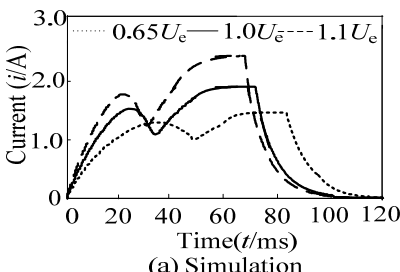

(a) Simulation

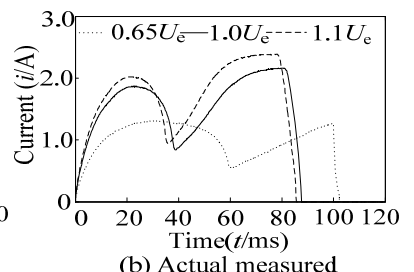

(b) Actual measured
FIGURE IV. THE SIMULATION AND ACTUAL MEASURED WAVEFORMS WHEN THE COIL VOLTAGE VARIES.

The simulation and actual measured waveforms of the CC when the coil resistance varies is shown in Figure V. As is shown in Figure $V$, the duration of stage $t_{0} \sim t_{1}$ is increased with the increase of the coil circuit resistance, which means that the inherent operation time of moving contact and energizing time of the coil is increased with the increase of the coil circuit resistance. Moreover, each feature of the current is reduced when the coil circuit resistance increases.

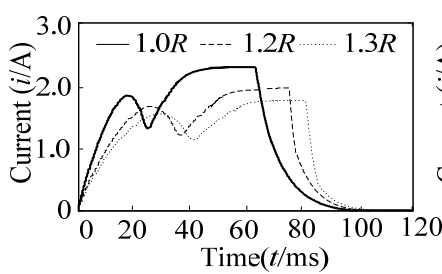

(a) Simulation

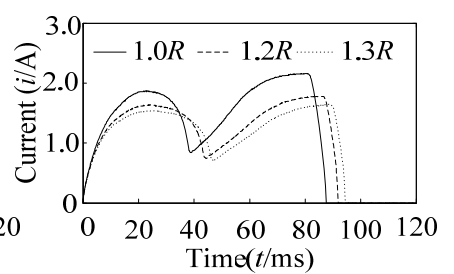

(b) Actual measured

\section{FIGURE V. THE SIMULATION AND ACTUAL MEASURED WAVEFORMS WHEN THE COIL RESISTANCE VARIES.}

The stimulation and actual measured waveform of the CC when the mechanism is in failure is shown in Figure VI. The figure shows that inflections appear at stage t2 t3. From the above analysis we can know, the current waveform at this stage can reflect the working condition of the transmission mechanism of switch, indicating that the transmission part of the main body is in fault, which is consistent with the condition of the actual fault.

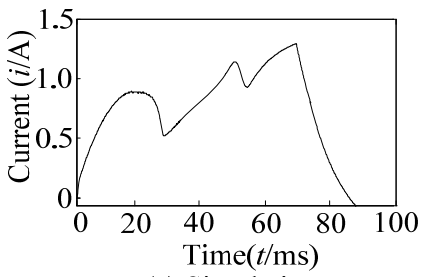

(a) Simulation

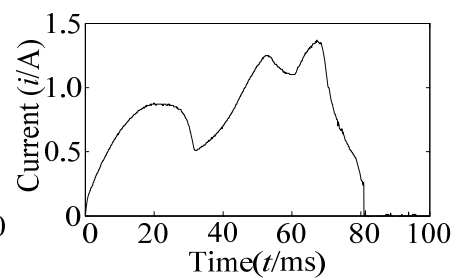

(b) Actual measured
FIGURE VI. THE STIMULATION AND ACTUAL MEASURED WAVEFORM WHEN THE MECHANISM IS IN FAILURE.

\section{CONDITION ASSESSMENT}

\section{A. Extraction of the Features and Inflections}

In a practical system, noise exists in the CC signal, so in 
this research the $\mathrm{CC}$ waveform is filtered first by the five points three times smoothing filtering method [18]. Then the slope of adjacent two points of the waveform is calculated to extract the features and inflections of the $\mathrm{CC}$ waveform according to the sign of the slope. Obviously, the point is a feature or inflection when the slope is 0 .

Assume that the collected CC signal is V[i]. The slope of each point on the time series can be expressed as follows:

$$
k_{i}=\left(V_{i+1}-V_{i}\right) / T, i \in[0, n]
$$

Where, $i$ is the sampling sequence number $(i=0,1, \cdots, n), T$ is the sampling period.

Figure VII presents the comparison of the CC waveform and the slope curve. As is shown in Figure VII, the crossing times correspond to the first, second, third and fourth slope curve crossing the $T$ axis are $t_{1}, t_{2}, t_{3}, t_{4}$, respectively. They and their corresponding current can be extracted easily. The corresponding current is obtained by the way of inquiry in this paper. By evaluating the total number of the extracted features and inflections to determine whether there is an inflection in the $\mathrm{CC}$ waveform, and the time position of the extracted point determines the point to be a feature or inflection.

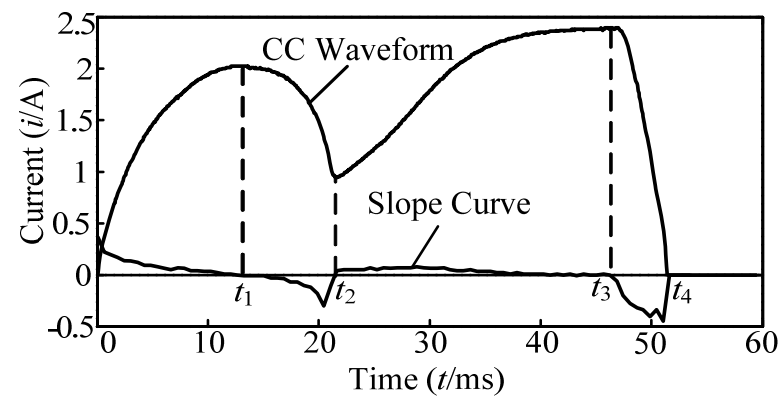

FIGURE VII. COMPARISON OF CC WAVEFORM AND SLOPE CURVE.

\section{B. Optimization of the Features}

From the analysis of section 1, we can know that the 8 features contain most of the key information of the $\mathrm{CC}$ waveform. However the 8 features contain much redundant information. In this subsection, the features are optimized to reduce the dimension and exclude highly relevant features.

Pearson correlation coefficient is employed to reveal the relationships of the features of measured data [16]. And it is used via (4) to explore the correlation of the parameters.

$$
r=\frac{\sum_{i=1}^{n}\left(X_{i}-\bar{X}\right)\left(Y_{i}-\bar{Y}\right)}{\sqrt{\sum_{i=1}^{n}\left(X_{i}-\bar{X}\right)^{2}} \sqrt{\sum_{i=1}^{n}\left(Y_{i}-\bar{Y}\right)^{2}}}
$$

Where $\left(X_{i}, Y_{i}\right), \bar{X}$ and $\bar{Y}$ are the paired data and the mean of dataset, respectively. $r \subseteq[-1,1]$, which describes the degree of linear correlation between two features. The greater the absolute value of $r$ is, the stronger the correlation is. The midpoint of its range, 0 , indicates a complete absence of correlation. Pearson correlation coefficient is generally considered to have significant correlation with the absolute value greater than 0.5 and have little correlation with the absolute value lower than 0.5 .

In this paper, the operation mechanisms of $40.5 \mathrm{kV}$ HVCBs are tested, and 35 groups of data during opening operation are recorded. The correlation between the 8 features for the 35 groups of data is analyzed via (4). Table I presents the results of the correlation index analysis for the 35 groups of data. (Usually $t_{0}=0$, thus $t_{0}$ is omit in Table I.) Worthy to note is that similar trends are observed during these investigations on features of the $\mathrm{CC}$ of closing operations.

As is shown in Table I, according to Pearson correlation coefficient, the features can be divided into three groups as follows: $\left\{i_{1}, i_{2}, i_{3}\right\},\left\{t_{1}, t_{2}, t_{3}\right\}$ and $\left\{t_{4}\right\}$. Therefore, $i_{1}, t_{1}$ and $t_{4}$ can represent the characteristics of the $\mathrm{CC}$ to the maximum extent. In this paper, $i_{1}, t_{1}$ and $t_{4}$ have been selected to develop a precise operating mechanism condition assessment method. And finally, the work of the calculation for the condition assessment will be greatly reduced.

TABLE I. CORRELATION INDEX FOR THE FEATURES

\begin{tabular}{c|c|c|c|c|c|c|c}
\hline & $\boldsymbol{i}_{\mathbf{1}}$ & $\boldsymbol{i}_{\mathbf{2}}$ & $\boldsymbol{i}_{\mathbf{3}}$ & $\boldsymbol{t}_{\mathbf{1}}$ & $\boldsymbol{t}_{\mathbf{2}}$ & $\boldsymbol{t}_{\mathbf{3}}$ & $\boldsymbol{t}_{\mathbf{4}}$ \\
\hline$i_{1}$ & 1 & 0.92 & 0.96 & 0.24 & 0.37 & 0.36 & 0.38 \\
\hline$i_{2}$ & 0.92 & 1 & 0.94 & 0.20 & 0.26 & 0.30 & 0.30 \\
\hline$i_{3}$ & 0.96 & 0.94 & 1 & 0.36 & 0.45 & 0.46 & 0.43 \\
\hline$t_{1}$ & 0.24 & 0.20 & 0.36 & 1 & 0.90 & 0.98 & 0.75 \\
\hline$t_{2}$ & 0.36 & 0.26 & 0.45 & 0.90 & 1 & 0.93 & 0.62 \\
\hline$t_{3}$ & 0.35 & 0.29 & 0.46 & 0.98 & 0.93 & 1 & 0.60 \\
\hline$t_{4}$ & 0.37 & 0.30 & 0.43 & 0.75 & 0.62 & 0.60 & 1 \\
\hline
\end{tabular}

C. Condition Assessment of Operating Mechanism Based on BP Neural Network and Waveform Recognition

Compared with the condition assessment method that used only the features of the $\mathrm{CC}$ waveform, this paper evaluates the operation state of the operating mechanism by the combination of features and inflections using the BP neural network and waveform recognition.

1) BP Neural Network: Back Propagation (BP) neural network can learn and store a large number of mapping relation between input and output, without prior knowledge of the mathematical equations that describe this mapping, with nonlinear mapping ability, self-learning ability and a good fault tolerance [17], [18].

The structure of BP neural network model is shown in Figure VIII, which includes input layer, hidden layer and output layer. The learning process of BP neural network is composed of forward and backward propagation. Forward propagation is used to calculate the network, obtaining the output of a certain input. Backward propagation is used to transfer error layer by layer, modifying the connection weights and thresholds. The process can be divided into 5 steps. 


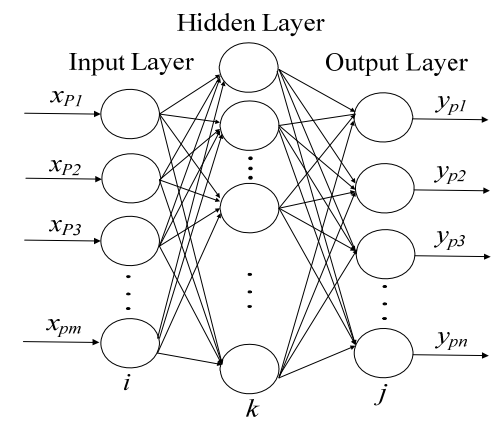

FIGURE VIII. STRUCTURE OF BP NEURAL NETWORK

Step 1, initialize the weights, thresholds and momentum factors of BP neural network.

Step 2, determine the structural parameters of BP neural network and give sample pairs of the input and output.

Step 3, compute input and output values of each node.

Step 4, update the weights and thresholds of each layer according to the error.

Step 5, calculate the total error of neural network training. If the accuracy meets the requirements, the training will end. Otherwise, go back to Step 2 and begin a new round of learning.

2) Waveform Recognition: Waveform recognition assesses the condition of equipment through the comparison of the current waveform with the standard waveform in the sample library. This method is simple and easy to realize, which can quickly come to the conclusion of the first for the experienced technical personnel.

Figure IX shows the process of the waveform recognition. Waveform recognition can assess the condition of operating mechanism through judging whether there are inflections in the $\mathrm{CC}$ waveform apart from the features. However, the mechanism state evaluation method based on waveform recognition can not assess the condition of the mechanism when the features are changed.

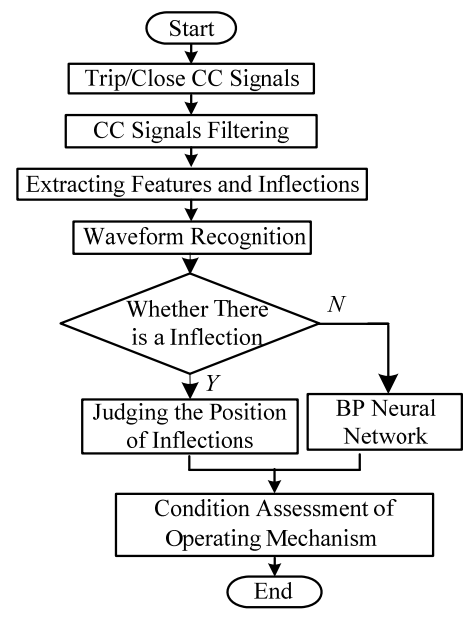

FIGURE IX. THE EVALUATION PROCESS BASED ON WAVEFORM RECOGNITION.
3) Comprehensive Evaluation Process of Operating Mechanism: Figure $\mathrm{X}$ presents the process of the method based on waveform recognition and neural network for evaluating the operating mechanism condition. First of all, the system assesses whether there is any other inflection in the $\mathrm{CC}$ waveform apart from the features by identifying the $\mathrm{CC}$ waveform of the measured mechanism. If there is a inflection, the system analyze the position of the inflection to evaluate the running state of the mechanism. If there is no inflection, then the system analyzes the features by BP neural network to evaluate the running state of the mechanism.

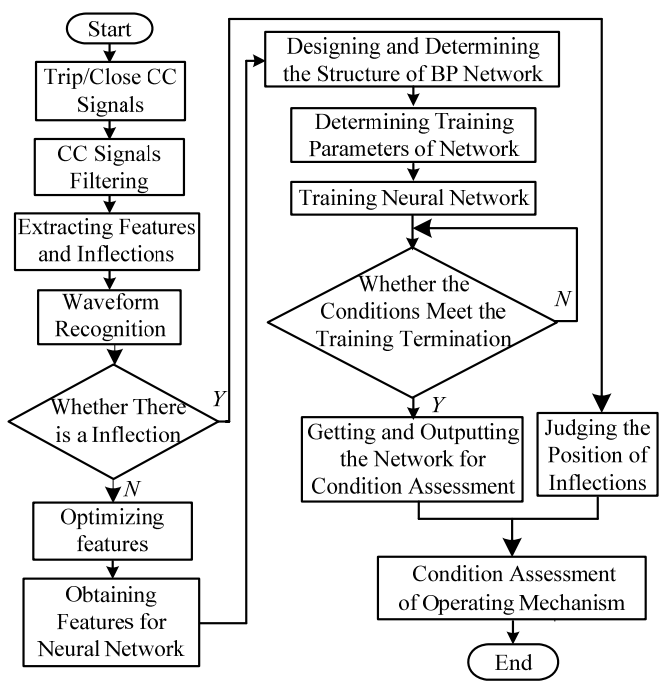

FIGURE X. THE EVALUATION PROCESS BASED ON BP NEURAL NETWORK AND WAVEFORM RECOGNITION.

\section{Case Study}

In this paper, $252 \mathrm{kV}$ spring operating mechanisms are tested, and the features and inflections of the CC waveform after filtering are extracted as the characteristic parameters of the operating mechanism state evaluation. Six types of operating mechanism state samples are selected to train and test the evaluation system, including normal state (A), trip coil voltage is too low (B), trip coil voltage is too high (C), bad contact of the auxiliary contact (D), the main body of the operation mechanism occurs jam during opening operation $(\mathrm{E})$, iron core air travel is too long (F). Each type of the sample for training and testing has 10 groups and 2 groups of data, respectively. Test samples are shown in Table II. I, II and III in Table II, respectively, indicate $t_{0} \sim t_{2}, t_{2} \sim t_{3}$ and $t_{3} \sim t_{4}$ stages of the $\mathrm{CC}$ waveform. 0 indicates that there are no inflections, 1 indicates that there are inflections.

The evaluation model is tested by the test samples in Table II to obtain the evaluation results of the operating mechanism. The results are shown in Figure XI. As is shown in Figure XI, the evaluation model can make a correct classification of all test samples, and the output value is stable. Obviously, the output result of the system is in good agreement with the actual operation conditions of the operating mechanism, which indicates that the evaluation model established in this paper can evaluate the operation state of the operating mechanism accurately. 
TABLE II. TEST SAMPLES OF TRIP CC FEATURES AND INFLECTIONS

\begin{tabular}{|c|c|c|c|c|c|c|c|}
\hline Number & $\overline{i_{1} / \mathbf{A}}$ & $t_{1} / \mathrm{ms}$ & $t_{4} / \mathbf{m s}$ & I & II & III & State \\
\hline 1 & 1.62 & 24.57 & 50.02 & 0 & 0 & 0 & $\mathrm{~A}$ \\
\hline 2 & 1.61 & 24.51 & 50.30 & 0 & 0 & 0 & A \\
\hline 3 & 1.23 & 33.96 & 59.08 & 0 & 0 & 0 & B \\
\hline 4 & 1.29 & 32.10 & 57.00 & 0 & 0 & 0 & $\mathrm{~B}$ \\
\hline 5 & 1.86 & 24.28 & 45.39 & 0 & 0 & 0 & $\mathrm{C}$ \\
\hline 6 & 1.79 & 24.25 & 46.10 & 0 & 0 & 0 & $\mathrm{C}$ \\
\hline 7 & 1.60 & 24.93 & 50.01 & 0 & 0 & 1 & $\mathrm{D}$ \\
\hline 8 & 1.62 & 24.76 & 49.98 & 0 & 0 & 1 & $\mathrm{D}$ \\
\hline 9 & 1.60 & 24.12 & 50.02 & 0 & 1 & 0 & $\mathrm{E}$ \\
\hline 10 & 1.60 & 24.55 & 49.99 & 0 & 1 & 0 & $\mathrm{E}$ \\
\hline 11 & 1.71 & 27.88 & 54.20 & 0 & 0 & 0 & $\mathrm{~F}$ \\
\hline 12 & 1.80 & 29.91 & 55.11 & 0 & 0 & 0 & $\bar{F}$ \\
\hline 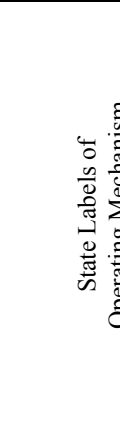 & $A_{1}$ & $\begin{array}{r}\text { Predic } \\
\text { Actu }\end{array}$ & $\begin{array}{l}-1-- \\
\text { State } \\
\text { tate }\end{array}$ & $\begin{array}{l}i \\
1 \\
\end{array}$ & i & & \\
\hline
\end{tabular}

FIGURE XI. THE EVALUATION RESULTS OF OPERATING MECHANISM.

\section{CONCLUSiOnS}

This paper has analyzed the relationship between the $\mathrm{CC}$ waveform and the operation state of the HVCB operating mechanism. The influence of the mechanical parameter variations and mechanism faults on the $\mathrm{CC}$ waveform has been studied through simulation and practical experiments. The simulation model is established by the FEM which has higher accuracy compared to the magnetic circuit method. Finally, the condition of the HVCB operating mechanism is evaluated by the combination of the waveform recognition and neural network analysis, and the following conclusions are drawn:

1) Trip/close CC waveform is closely related to the running state of the operating mechanism. The simulation results are consisted with the practical test results, which proves that the features and inflections of the $\mathrm{CC}$ waveform can be used as the accurate evaluation indices of the operating mechanism.

2) This paper uses the slope method to extract the features and inflections of the $\mathrm{CC}$ waveform after filtering. And the features are optimized by Pearson correlation coefficient to remove the redundant information between features, which avoids the "curse of dimensionality" because of too many inputs.

3) The method based on the combination of the waveform recognition and neural network for evaluating operating mechanism state not only considers the change of the features of the CC waveform, but also takes the change of the waveform itself. The evaluation results can reflect the operation state of the HVCB operating mechanism more accurately.

\section{REFERENCES}

[1] A.A Razi-Kazemi, "Applicability of auxiliary contacts in circuit breaker online condition assessment," Electric Power Systems Research, vol. 128, pp. 53-59, 2015.

[2] A. Janssen, D. Makareinis, and C. E. Solver, "International surveys on circuit breaker reliability data for substation and system studies," IEEE Trans. Power Delivery, vol.29, no.2, pp. 808-814, Apr. 2014.

[3] W. Guo, F. Wen, G. Ledwich, Z. Liao, X. He, and J. liang, "An analytic model for fault diagnosis in power systems considering malfunctions of protective relays and circuit breakers," IEEE Trans. Power Delivery, vol. 25, no. 3, pp. 1393-1401, 2010.

[4] Working Group A3.12, "Failure survey on circuit breaker control systems summary report for Electra," CIGRE, Electra no.216, Oct 2004.

[5] M. Runde, "Failure frequencies for high-voltage circuit breakers, disconnectors, earthing switches, instrument transformers, and gasinsulated switchgears," IEEE Trans. Power Delivery, vol.28, no.1, pp. 529-530, Jan.2013.

[6] B. Stephen, S.M. Strachan, S.D.J. Mcarthur, J.R. Mcdonald, "Design of trip current monitoring system for circuit breaker condition assessment," IET Generation Transmission \& Distribution, vol.1, no.1, pp. 89-95, 2007.

[7] A. A. Razi-Kazemi, M. Vakilian, K. Niayesh, and M. Lehtonen, "Priority assessment of online monitoring investment for power system CBs part II: determination of optimum number," IEEE Trans. Power Delivery, vol. 29, no. 2, pp. 1440-1446, 2015.

[8] A. A. Razi-Kazemi, M. Vakilian, K. Niayesh, M. Lehtonen, "CircuitBreaker automated failure tracking based on coil current signature," IEEE Trans. Power Delivery, vol.29, no.1, pp. 283-290, Feb. 2014.

[9] M. Andruşcă, M. Adam, R. Pantelimon, and A. Baraboi, "About diagnosis of circuit breakers," in Electrical Engineering 2013 International Symposium on Advanced Topics, pp. 1-4.

[10] A. Forootani, A. A. Afzalian, and A. Melli, "Circuit breaker coil modeling and operation monitoring using feature extraction," in 2012 Innovative Smart Grid Technologies- Asia , pp. 1-6.

[11] C.Y. Cheng, L. J. Chen, and W. S. Kao, "Diagnosing medium voltage GIS circuit breaker by observing trip coil current with simulation and actual tests," Electric Power Components \& Systems, vol. 36, no. 2, pp. 181-194, 2008.

[12] Y. Zheng, "A Model-Based Fault Detection Framework for Vacuum Circuit Breaker by Trip Coil Analysis," Journal of Software, vol. 9, no.1, pp. 251-256, 2014.

[13] J. Ni, C. Zhang, and S. X. Yang, "An adaptive approach based on KPCA and SVM for real-time fault diagnosis of HVCBs," IEEE Trans. Power Delivery, vol.26, no.3, pp. 1960-1971, 2011.

[14] S. Ito, Y. Takato, Y. Kawase, and T. Ota, "Numerical analysis of electromagnetic forces in low voltage AC circuit breakers using 3-D finite element method taking into account eddy currents," IEEE Trans. Magnetics, vol. 34, no.5, pp. 2597-2600, 1998.

[15] A. Abri, R. Nordgren, S. Kjellnas, and L. Banghammar, "Finite element analysis of electromagnets and contact systems in low voltage current limiting circuit breakers," IEEE Trans. Magnetics, vol. 113, no.2, pp. 960-963, 1990.

[16] A. A Razi-Kazemi, "Circuit breaker condition assessment through a fuzzy-probabilistic analysis of actuating coil's current," IET Generation Transmission \& Distribution, vol. 10, no.1, pp. 48-56, 2016.

[17] R.Mingzhe,W. Xiaohua, Y.Wu, and J. Shenli, "Mechanical condition recognition of medium-voltage vacuum circuit breaker based on mechanism dynamic features simulation and ANN," IEEE Trans. Power

[18] Delivery, vol. 20, no. 3, pp. 1904-1909, Jul. 2005.

[19] B.S. Qi, "Research on fault diagnosis of high-voltage circuit breaker based on the improved BP neural network," in 2010 IEEE Information Science and Engineering (ICISE), Conf., pp.1460-1463. 